\title{
Vol. 69, No. 43
}

In the report "COVID-19-Associated Hospitalizations Among Health Care Personnel - COVID-NET, 13 States, March 1May 31, 2020," on page 1579, in the Table, the row headings for rows 13 and 14 were incorrect and should have appeared as below:

$\begin{array}{llcccc}\text { Asian or Pacific Islander, non-Hispanic } & 39(6.8) & (4.2-10.8) & 29(6.8) & (4.0-11.5) & 10(6.7) \\ \text { American Indian or } & 12(3.2) & (1.5-6.6) & 10(4.4) & (2.0-9.6) & 2(0.6)\end{array}$

Alaska Native, non-Hispanic

\section{Vol. 69, No. 43}

In the report "Trends in the Use of Telehealth During the Emergence of the COVID-19 Pandemic - United States, January-March 2020," on page 1595, a name in the list of authors was incorrect. The author's name should have read B. Tilman Jolly. In addition, on page 1597, a comma was mistakenly included in the phrase 1,135 waivers. The phrase should have read " 1135 waivers." 